\title{
Effection of Current Transformer's Accuracy Error on Current Differential Protection
}

\author{
Jing Hua ${ }^{1, \mathrm{a}}$, Li $\mathrm{Al}^{2, \mathrm{~b}}$ and Jiatang Cheng ${ }^{3, \mathrm{c}}$ \\ The Engineering College of Honghe University, Yunnan Mengzi 661199

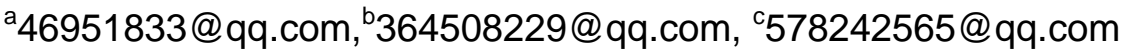

Keywords: Current transformer; Differential protection; Saturation; Current of each string

\begin{abstract}
In order to improve the accuracy of the current differential protection, we can detect from the current transformer which can collect reference amount and transfer current. By analysis of the equivalent circuit of the current transformer, the saturation current waveform, the saturation current of harmonic waveform and current transformer secondary circuit shunt of single-phase ground fault.
\end{abstract}

\section{Introduction}

The principle of current differential protection is based on Kirchhoff's law, it has good selectivity, can be sensitive and fast to remove the fault in the protection area, and being widely used in the protection of generator, transmission line, transformer, bus and so on, which can easily get the terminal current ${ }^{(1)}$.

Current transformer (CT) is an important measuring element in power system. However, due to the nonlinear and hysteresis characteristics of CT core, when the large fault current to make CT work in the area of nonlinear excitation characteristics, CT into saturation, the second current distortion, can not accurately transfer the first current, resulting in a transmission error. Due to CT saturation caused transmission error brought very adverse effects on relay protection, especially the current differential protection which is widely used, even causes disoperation of differential protection, seriously affected the further enhance the protection of the accuracy, therefore, we must solve the problem of saturation of CT.

For CT saturation, the general use of the ratio of braking characteristics to overcome. However, the reliability of differential protection can not be guaranteed under the condition of extreme saturation of CT by using ratio braking only, and it is necessary to use other methods to identify CT saturation. 。 At present, the main method of CT saturation identification is differential method ${ }^{\text {[3] [4] }}$. In addition, a new method based on mathematical morphology is proposed to identify the saturation of $\mathrm{CT}^{[8]}$, which uses mathematical morphology to extract the characteristics of the current waveform after CT saturation to distinguish the internal fault and external fault of CT saturation.

\section{Influence of CT Saturation Current on Differential Protection}

CT Saturation Current Analysis. The second side load impedance angle of CT will cause the secondary current waveform distortion. In order to take into account the two factors, the current waveform of the first or the secondary side of the current transformer. When the first current is in a steady state, as shown in Fig. 1 a and c, the non periodic content in the primary current is zero, and the secondary current in the positive and negative half cycles is saturated; When the first current is transient, as shown in Fig. b, d shows, the first non periodic current content is $100 \%$, primary current biased to one side of the time axis, CT only in a half cycle saturation occurs, this is called asymmetric saturation. When CT saturation, it will appear the following characteristics

1. The occurrence of faults and the existence of a certain time difference in the saturation of CT;

2. The secondary current waveform defects and distortion, which is equivalent to the power frequency current superimposed on the harmonic, the secondary and the third harmonic is the main harmonic component;

3. The secondary current in the saturation point and the point of retreat will be a mutation;

4. The secondary current value is suddenly reduced, and the waveform is defective, especially 
when the saturation of CT is more serious, the output of the secondary current is almost zero.

Harmonic Analysis. The secondary current after CT saturation in a harmonic component to a certain extent, Fig. 2 is CT respectively, symmetry and non symmetry saturation (the second side load impedance $\varphi=0$ ) the fundamental and the first six harmonic maps. From the graph, we can see that the harmonic content of the CT is dominated by odd harmonics, especially the third harmonic is the largest, and even the second harmonic content is relatively small; and CT occurs when the asymmetry of saturation, harmonic content mainly to the second harmonic and the third harmonic, higher harmonic content is relatively low. In addition, whether it is symmetric or asymmetric, the amplitude of the fundamental current is larger than the amplitude of the harmonic current. Through the experimental analysis, it is known that the fundamental current component is still the main component in the saturation current. The large fault current will cause saturation resulting in saturated current transformer circuit, both the constant impedance load side or load side with electric motor, the saturation current will bring the harmonic in different degree, the saturated current effects that leads to errors, lead to disoperation of circuit breaker.

\section{Effect of Alternating Current on Differential Protection}

Equivalent Circuit of Current Transformer. The equivalent model of the current transformer is shown in Fig. 3. It can be seen from the diagram that the primary side can be regarded as a current source in parallel with a nonlinear inductance. In normal operation, the excitation impedance is very large, the excitation current is very small, but when the transformer saturation
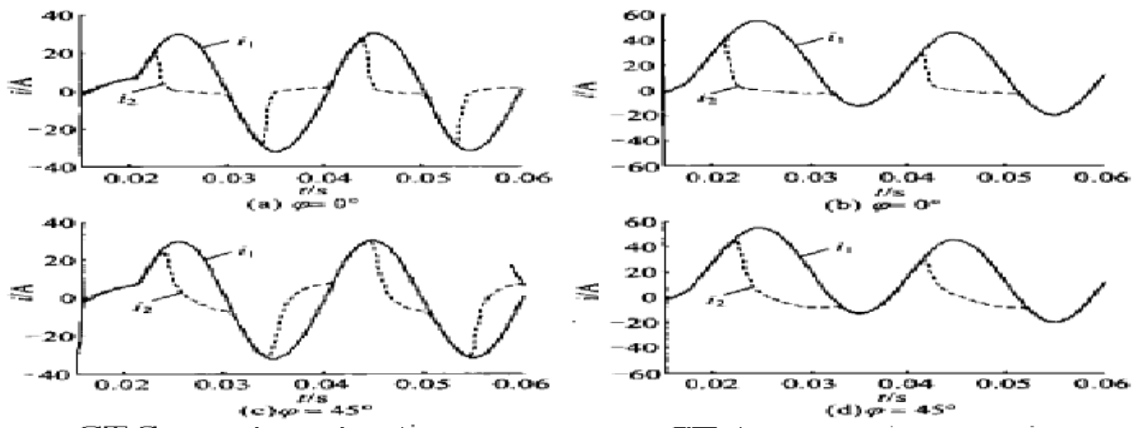

CT Symmetry saturation

CT Aspmmetric saturation

Figure 1. CT saturation current waveform

$L_{b}$ small, the excitation impedance of $\omega L_{b}$ is small; Similarly, when the current contains a non-periodic component $\omega=0$, equivalent to the excitation branch reactance is changed to 0 , the first current all injected into the excitation branch, the secondary current is close to 0 .

The Reason of the Current String. In the actual system, the secondary circuit of the current transformer for protection is shown in Fig. 4. We can see from Fig. 4, in the secondary loop connection mode of actual, for the A phase current transformer secondary winding is two, the second phase of the load resistance and the A, B, C three-phase public resistance $\mathrm{N}$ of line.

When Disoperation happen, the common features is non fault phase currents' polarity always contrary to the fault phase currents', which can make a preliminary judgment: non fault phase currents is generated by current transformer's secondary circuit which diverted to non fault by fault phase current. As shown in Fig. 4: set the fault phase for the A phase, A phase secondary current through the A phase of the secondary device current inflow into the secondary circuit of transformer. Based on the above judgment, the main features of the current can be analyzed:

1) The burr current is generated from the fault phase to the non fault phase, and the non fault phase is opposite to the fault phase.

2) The fault current inflows into the non fault phase (especially the non periodic component), which leads to the non fault phase current transformer saturation.

3) The current of the fault phase is generally not possible to continue to the non fault phase in series, the fault phase of the current acts as a non fault phase to the magnetic current, so the duration 
of the burr current is usually shorter.

4) The burr current caused by the non fault phase current transformer saturation is very large, which may cause the differential protection of the bus, the start of the line differential protection and even the malfunction.

\section{Conclusions}

In this paper, through theoretical analysis, which discusses two methods of precise effect of differential protection, for the first kind of influence factors, transformer saturation circuit and harmonic through different discriminate saturation current and
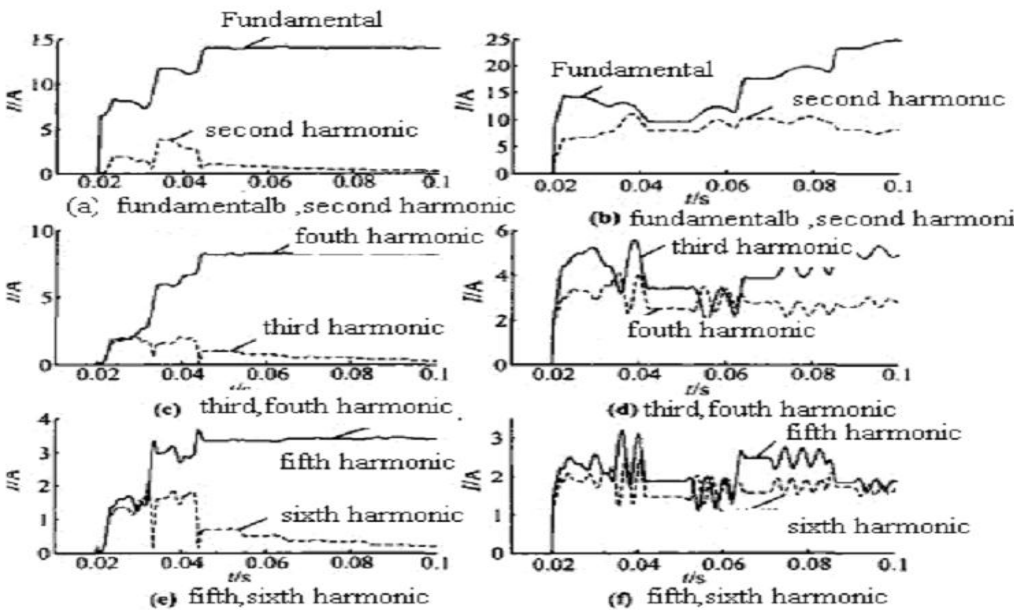

CT Symmetry saturation

CT Asymmetric saturation

Figure. $2 \mathrm{CT}$ saturation current harmonics

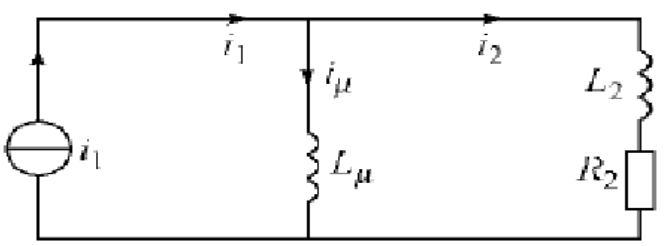

Figure. 3 equivalent cir cuit of current transformer

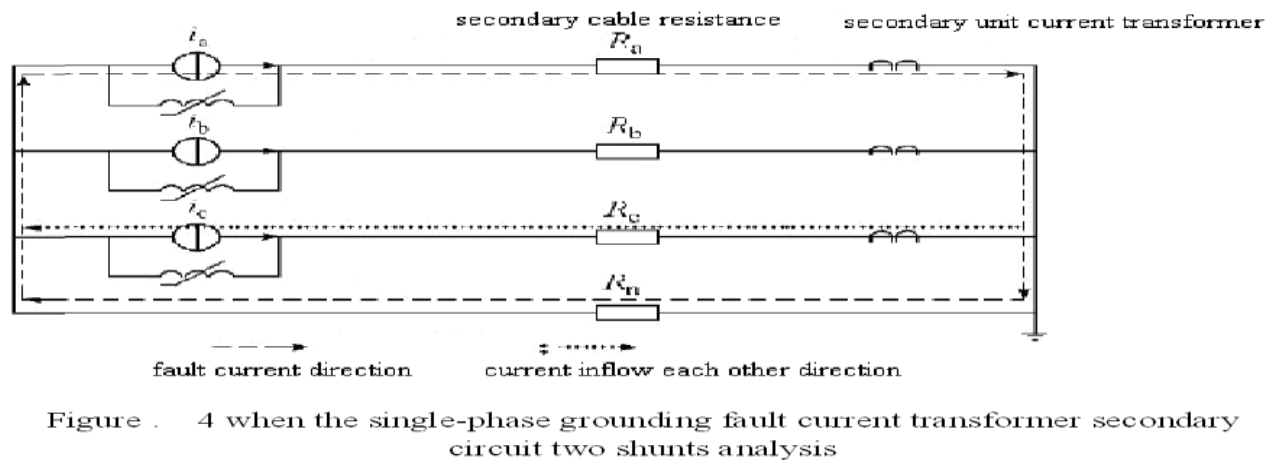

fault current to prevent disoperation, in distinguishing saturated current protection or artesian, escaped in saturation current differential protection setting calculation; For second kinds of influencing factors, the fault initiation and differential current initiation of the non fault phase is initiated at the same time, the current transformer saturation criterion is more difficult to solve the problem, which needs to be paid attention to. With the expansion of power grid scale, the short circuit capacity of the power network becomes larger and larger, which may occur frequently. According to the analysis of this paper, we need to focus on the following issues:

1) The saturation characteristics of current transformer for measurement, combined with the 
secondary loop resistance is calculated, in the judgment of the short-circuit capacity of the current situation, whether there will be the second current shunt phenomenon also, caused by the incorrect action of protection phenomenon.

2) Need to pay attention to the connection of the secondary circuit neutral point, whether there is a phenomenon of poor contact or insulation damage.

3) Saturation of current transformer is a problem to be concerned. For the current protection with the current transformer, in the equipment selection should be a good off, in strict accordance with the protection of current transformer technical conditions for selection, we should replace the current transformer which does not meet the conditions. Preferred low remanence current transformer, and improve the inflection point saturation of current transformer, increase capacity and ratio, reduce the secondary loop resistance ratio, especially resistance of neutral $\mathrm{N}$ line ( $\mathrm{N}$ line should be increased gradually, the number of parallel cable) and improve the second loop.

4) A new protection algorithm is studied, such as the fault phase current is introduced from the non fault phase as the braking capacity, the addition of voltage criterion and so on.

\section{References}

[1] Bao-hui Zhang, Xiang-gen Yin, Power System Protection Relay. BeiJing: China Electric Power Press2005

[2] Yu-bo Yuan Research on the principle and method of adaptive digital transformer differential protection [D]. NanJing: east-south college, 2003.

[3] Yan-Li De-shu Chen Zhe Zhang etc. Study on the improved time difference method for the identification of current transformer saturation. Protection,2001, 29(11): 1-4

[4] Sheng Jing, Xiang-yang Liu. Analysis of the error caused by the differential protection of the busbar differential protection caused by the saturation of the converter. Protection, 2004, 32(2): 70-71.

[5] Xiao-bing Ding Man-yong Zhao Xian-song Pi etc. Measures to prevent the malfunction of busbar protection caused by AC series DC to DC bus. Power system protection and control, 2008, 36(22): 97-99

[6] Ji-Xiu Yuan,He-Le Sheng, Ju-ye Wu. Guide for application of current transformers for protection [M]. BeiJing: China Electric Power Press, 2004 [J] . Automation of electric power system, 2000, 24(6):54-56

[7] Jian-Yu Chen,Xian-min Meng, Zheng-qi Zhang etc Analysis and Countermeasures of the influence of saturation of current transformer on relay protection. Protection, 2008, 34(3): 87-89.

[8] Yan Li, De-shu Chen, Zhe Zhang etc. Study on improved time difference method for identification of TA saturation [J]. Relay, 2010, 29(11): 1-5

[9] Bo-Fang Zhu Finite Element Method Theory and Application [M]. China Water Power Press, 1998

[10]Qing-He Qi. Hydraulic Structures [M]. China Water Power Press, 2009 